\title{
Diversity of sex-determining alleles in Bracon hebetor
}

\author{
GEORGE E. HEIMPEL*†, MICHAEL F. ANTOLIN $\uparrow \&$ MICHAEL R. STRAND $\dagger$ \\ $\uparrow$ Department of Entomology, University of Wisconsin, Madison, WI 53706, U.S.A. and $\ddagger$ Department of Biological \\ Sciences, Colorado State University, Fort Collins, CO 80526, U.S.A.
}

\begin{abstract}
In many hymenopterans, sex is determined at a single polymorphic 'sex locus'. Individuals that are heterozygous at this locus develop as females whereas homozygotes and hemizygotes develop as diploid and haploid males, respectively. Diploid males are developmentally inviable or sterile, and the likelihood of diploid male production depends in large part on allelic diversity at the sex locus. We assessed sex allele diversity within and among five U.S. populations of the parasitoid wasp Bracon hebetor using a series of crosses between isofemale lines. The study included two laboratory populations originating in Wisconsin, two field populations originating in Kansas and California, and a population purchased from a commercial insectary. Given the number of isofemale lines that we established, the maximum number of alleles that we could detect per population was 10 . The number of sex alleles identified within populations ranged between three or four (for the two Wisconsin populations) and nine (for the California population). Subsampling three or four alleles from each population for between-population crosses led to identification of 12 alleles. Of these, four were unique to the California population, three were unique to one other population each, and one was found in only two populations. Extrapolation of the relationships between the subsampled lines led to a total estimate of 20 alleles within our lines. The relatively high allele diversity in the field and commercial insectary populations suggests that the sex determination load is relatively low in $B$. hebetor, and the differences in allele profiles between populations suggest that interpopulation dispersal can increase sex allele diversity within populations.
\end{abstract}

Keywords: Bracon hebetor, complementary sex determination, diploid males, Hymenoptera, sex alleles, sex ratio.

\section{Introduction}

In most animals, sex is determined chromosomally, with either the specific combinations of sex chromosomes or the ratio of sex chromosomes to autosomes determining sex. In some arthropods and rotifers, however, sex determination is linked to fertilization (Bull, 1983). Under haplo-diploidy, unfertilized eggs develop as haploid males and fertilized eggs typically develop as diploid females. Among insects, most species in the order Hymenoptera have haplo-diploid sex determination. Nonetheless, some species of Hymenoptera produce diploid males through a mechanism known as 'single-locus complementary sex determination' (henceforth CSD). Under CSD, the sex of fertilized eggs is determined at a single polymorphic locus (Cook, 1993b). Eggs that are heterozygous at the 'sex locus' develop as

*Correspondence and present address: Department of Entomology, University of Minnesota, St. Paul, MN 55108, U.S.A. E-mail: heimp001@tc.umn.edu diploid females, whereas homozygotes develop as diploid males. Diploid males are either developmentally inviable or sterile and their production constitutes a genetic load associated with CSD (Werren, 1993), the severity of which depends critically on the number of sex alleles within and among populations (Cook \& Crozier, 1995). Stouthamer et al. (1992) showed that the sex determination load is strongest when the number of sex alleles per population drops below six. Sex allele diversity is generally inferred from the frequency of females in a population that produce diploid males (Owen \& Packer, 1994) and within-population estimates of the number of sex alleles among various species range between 10 and 86 (Cook \& Crozier, 1995). The levels of polymorphism observed at the hymenopteran sex locus therefore rival those found in genes from the major histocompatability complex in mammals and self-incompatibility loci in plants (Potts \& Wakeland, 1993).

Despite the great potential for sex allele diversity within populations, the extent to which sex alleles are 
shared or different among populations is unknown. One reason for this is that the most common ways of measuring sex allele diversity within populations, the frequency of diploid males or the frequency of females which produce diploid males, cannot be used for measuring sex allele divergence between populations. Instead, controlled laboratory crosses need to be carried out that distinguish between individual sex alleles. Here, we assess within- and between-population sex allele diversity in the parasitoid wasp Bracon hebetor (Hymenoptera: Braconidae), the first species in which sex determination was shown to be regulated by a series of alleles segregating at a single locus (Whiting, 1943). Our goals were to (i) determine whether within-population sex allele diversity is so low as to introduce a strong sex determination load (i.e. below six alleles per population), and (ii) assess the extent to which alleles are shared between populations. In the original work by Whiting, a series of crosses identified a total of nine alleles from six populations across the United States, with only two alleles identified in each population and a number of alleles shared between populations. Our analysis of five $B$. hebetor populations revealed that allele diversity can be high even in relatively small populations, and that some alleles appear to be shared across populations that are geographically isolated.

\section{Materials and methods}

\section{Natural history of Bracon hebetor}

Bracon hebetor is a Holarctic parasitoid of larvae of stored-food infesting moths in the pyralid subfamily Phycitinae (Krombein et al., 1979). The first U.S. records of B. hebetor are from Indiana (Say, 1836) and California (Johnson, 1895), and it is currently present throughout the U.S. and sold commercially as a biological control agent (Brower et al., 1996). A primary host for B. hebetor is the Indian meal moth, Plodia interpunctella, which infests stored grains, nuts and fruits (Brower et al., 1996). Bracon hebetor females inject a paralysing venom into mature $P$. interpunctella larvae, and then deposit between three and 20 eggs on the outside of the host (Benson, 1973). The egg hatch rate is typically greater than $90 \%$ for females and haploid males (Benson, 1973; Ode et al., 1996) and $\approx 10 \%$ for diploid males (Horn, 1943; Whiting, 1943; Grosch, 1945; Petters \& Mettus, 1980). The few diploid males that reach adulthood readily mate, but are almost all sterile (Whiting, 1961). Bracon hebetor females typically fertilize approximately two-thirds of their eggs, leading to female-biased brood sex ratios when sex alleles are not shared by the parents (e.g. Antolin et al., 1995; Heimpel et al., 1997; Ode et al., 1997).
Adult $B$. hebetor engage in a number of behaviours that facilitate outbreeding within the stored-grain habitat (Antolin \& Strand, 1992; Ode et al., 1995; Guertin et al., 1996). One of these behaviours, the avoidance of mating between individuals that developed together on the same host, could lead to a degree of outcrossing that reduces the occurrence of shared-allele matings, because these individuals tend to be siblings (Ode et al., 1995). However, siblings developing on different hosts readily mate with each other (Ode et al., 1995), as do mothers and sons (Petters \& Mettus, 1980; Cook et al., 1994; see below), suggesting that sex alleles per se are not used as cues for inbreeding avoidance.

Movement rates between stored-grain habitats are unknown, but it is likely that populations are at least temporarily isolated from one another because of the small size of many grain storage facilities and the large distances between them. The mixing of $B$. hebetor populations probably occurs most regularly during commercial practices, for instance when grain originating at multiple sites is stored together in central repositories. Many on-farm storage sites that receive no grain from other sources retain $B$. hebetor populations for many years, however. Grain bins are often emptied every year and it is thought that $B$. hebetor persist in the tailings left behind. Populations associated with on-farm storage sites are therefore potentially subject to genetic bottlenecks via both isolation from other populations and population crashes.

\section{Bracon hebetor populations}

We used five populations of $B$. hebetor. Two originated from maize-storage facilities in Dane Co., WI, U.S.A, and were initiated with over 100 female parasitoids. One of these (WKE, for Waunakee, WI) was collected in 1990 at a farm adjacent to a large maize-storage facility that received shipments from a three-county area, and the other (MID, for Middleton, WI) was collected in 1993 at an on-farm maize-storage site that received maize only from surrounding fields. The third population (KS) was initiated in 1996 with 31 males and 18 females collected from a single wheat silo in Sumner Co., KS, U.S.A. that harboured a small population of $B$. hebetor and received wheat only from surrounding fields. The fourth population (CA) was initiated with 41 males and 23 females collected from a large fig-storage facility in Fresno, CA, U.S.A. in 1996. This site received dried figs from a number of different farms in the area, each of which harboured a population of B. hebetor ( $\mathrm{J}$. Johnson, pers. comm.). The fifth population (INS) was initiated with $\approx 500$ individuals purchased from a commercial insectary (BioFac Crop Care, Mathis, TX, U.S.A.) just prior to the experiment. The commercially 
reared colony had been maintained for 8 years in cages containing more than 700 individuals each at the time of the purchase (anonymous, pers. comm.), as is recommended for the maintenance of high sex-allele diversity (Stouthamer et al., 1992). To minimize the possibility of contamination between populations, parasitoids were housed within $6 \mathrm{~cm}$ plastic Petri dishes which were housed in groups of four within small boxes $(12 \times 12 \times 3 \mathrm{~cm})$ which were themselves housed in larger boxes $(26.4 \times 19.2 \times 9.5)$, each of which contained a different population. All the populations were housed in a single growth chamber at $27^{\circ} \mathrm{C}$ and a 16L:8D photoperiod.

\section{Establishing two-allele lines}

We established five two-allele isofemale lines from each of the five $B$. hebetor populations by mating previously unmated females to their haploid sons. Single females were allowed to oviposit as virgins, held at $4^{\circ} \mathrm{C}$ until their sons emerged, and mated with these sons. These shared-allele matings resulted in two-allele lines that were maintained as populations of between 20 and 100 individuals throughout the experiments. The lines were housed in four $6 \mathrm{~cm}$ diameter Petri dishes, which were re-initiated twice weekly with five 3-7-day-old females and 10 mature $P$. interpunctella larvae as hosts. With this design, the maximum number of alleles sampled per population was 10. Although some populations may harbour more than 10 alleles (Whiting, 1943; Cook \& Crozier, 1995), the population-level consequences of the sex determination load are most severe when the number of alleles drops below six (Stouthamer et al., 1992).

\section{Crosses between lines}

We conducted a minimum of five replicate crosses for each combination of lines within populations (including within-line crosses as a control), and for a subsample of at least two lines per population for crosses between populations. For each group of five crosses, we set up two crosses in one direction and three in the reciprocal direction. Crosses were initiated by isolating mature pupae from the lines, and pairing single males and females in $6 \mathrm{~cm}$ Petri dishes at $27^{\circ} \mathrm{C}$ for $4 \mathrm{~h}$ between 2 and 4 days after adult emergence.

In order to determine which crosses resulted in shared-allele matings, we scored egg production, egg hatch, survivorship to adulthood, and secondary sex ratio (proportion of adult offspring that were male) for each female. Each day after mating, females were offered two mature $P$. interpunctella larvae that had been previously paralysed by a different female. These hosts had been exposed to $B$. hebetor females for $45 \mathrm{~min}$ earlier that same day, and were checked to ensure that no parasitoid eggs were present $(B$. hebetor females generally do not oviposit for $1-2 \mathrm{~h}$ after paralysing a host; Hagstrum \& Smittle, 1978). Approximately $24 \mathrm{~h}$ after exposure to hosts, we counted the number of eggs laid. The eggs were then distributed among additional paralysed hosts with a fine probe so that each host had no more than three eggs. This density of eggs/host minimized competition between parasitized larvae (Benson, 1973; Ode et al., 1996). Each female was given two hosts/day in this manner until she had laid at least 40 eggs, a process that usually took $3-5$ days. We scored egg hatch by counting the number of larvae present 34 days after oviposition. Sex ratio was scored $\approx 3$ weeks after oviposition, when all adult emergence had taken place. Crosses that produced only males were repeated with new males and females from the same lines.

\section{Identification of sex alleles}

We used the egg hatch and sex ratio data to classify each mating as being consistent with the parents either sharing or not sharing a sex allele. Typical characteristics of a mating in which sex alleles are not shared include an egg hatch rate exceeding $90 \%$ and a sex ratio of $\approx 0.33$ (proportion males). Shared-allele matings generally produce egg hatch rates below $65 \%$ and secondary sex ratios near 0.5 because $\approx 90 \%$ of male diploid progeny die as eggs (Horn, 1943; Whiting, 1943). These values are not constant across matings, however, because they are dependent on three parameters that vary among individual wasps. These are: (i) the fraction of eggs fertilized by ovipositing females; (ii) the egg mortality rate not attributable to male diploidy; and (iii) survivorship of diploid males to the adult stage. To incorporate these sources of variability, we developed a statistical test for classifying matings that is independent of a priori assumptions of the fertilization rate and takes into account variability in the background egg mortality rate and the survival of diploid males.

We begin by noting that the fertilization rate, $f$, of a shared-allele mating is $2 m$, where $m$ is the fraction of eggs not hatching, assuming that all diploid males die as eggs and all female and haploid male eggs hatch. To include additional mortality, the predicted fertilization rate can be expressed as $f^{*}=2\left(m_{\mathrm{obs}}-m_{\text {back }}\right)$, where $m_{\text {obs }}$ is observed egg mortality and $m_{\text {back }}$ is the background egg mortality experienced by haploid male and female eggs. The background mortality can be divided into female and haploid male components using the sex-specific mortality rates $\mu_{\mathrm{d}}$ (for females) and $\mu_{\mathrm{s}}$ (for haploid males):

$$
m_{\text {back }}=\left(\mu_{\mathrm{d}} f\right) / 2+\mu_{\mathrm{s}}(1 \quad f) .
$$


Expressed only in terms of the male mortality rate, this becomes:

$m_{\text {back }}=\left(a \mu_{\mathrm{s}} f\right) / 2+\mu_{\mathrm{s}}(1 \quad f)$

where $a=\mu_{\mathrm{d}} / \mu_{\mathrm{s}}$. This form of the equation is preferable because the parameter $a$ is available from the literature (Ode et al., 1996), and an independent measure of $\mu_{\mathrm{s}}$ is available from an analysis of matings that produced only male offspring.

The fertilization rate, $f^{*}$, is estimated from the proportion of surviving offspring that are female. This proportion decreases if some diploid males survive and a correction factor must be introduced to balance this effect. Incorporating the rate of diploid male survivorship, $\delta$, leads to

$$
\begin{aligned}
& f^{*}=2\left\{m_{\mathrm{obs}} \quad \mu_{\mathrm{s}}\left[\left(a f^{*} / 2\right)+1 \quad f^{*}\right]\right\} /\left(\begin{array}{ll}
1 & \delta
\end{array}\right) \\
& =2\left(m_{\mathrm{obs}} \quad \mu_{\mathrm{s}}\right) /\left[\mu_{\mathrm{s}}\left(\begin{array}{ll}
a & 2
\end{array}\right)+\left(\begin{array}{ll}
1 & \delta
\end{array}\right)\right] .
\end{aligned}
$$

This predicted fertilization rate can be used to calculate the predicted secondary sex ratio (proportion of adults that are males), $r^{*}$, under the assumption of shared sex alleles:

$$
\begin{aligned}
& r^{*}=\left[\left(\begin{array}{ll}
1 & f^{*}
\end{array}\right)+0.5 f^{*} \delta\right] /\left[\left(\begin{array}{ll}
1 & f^{*}
\end{array}\right)+0.5 f^{*} \delta\right. \\
& \left.+0.5 f^{*}\right] \\
& =\left[\begin{array}{lll}
1 & f^{*}(1 & 0.5 \delta
\end{array}\right] /\left[\begin{array}{lll}
1 & 0.5 f^{*}(1 & \delta
\end{array}\right),
\end{aligned}
$$

which can in turn be used to calculate the number of sons and daughters expected from a given cross producing $N$ adult offspring under the assumption of shared alleles. For each cross, we compared these values to observed numbers of adult male and female offspring produced using a log-likelihood goodness-of-fit test (Sokal \& Rohlf, 1981), which evaluates the null hypothesis that the pattern of egg mortality and sex ratio observed are consistent with a shared-allele mating.

We incorporated potential variation in background mortality, $\mu_{\mathrm{s}}$, and diploid male survivorship, $\delta$, by varying these parameters between low, central and high levels and running nine goodness-of-fit tests using all combinations of the values for each individual cross. Levels of background mortality were estimated from observed mortality rates of eggs laid by females that produced only males in the experiment (mean mortality rate $=0.13 \pm 0.09[\mathrm{SD} ; N=54]$; see below). Eggs produced by these females were presumably all haploid males and the fraction not hatching gave an estimate of $\mu_{\mathrm{s}}$. Low, central and high estimates of $\mu_{\mathrm{s}}$ were 0 , mean $\mu_{\mathrm{s}}$ $(=0.13)$ and (mean $\mu_{\mathrm{s}}+3 \times$ the standard deviation of $\left.\mu_{\mathrm{s}}=0.40\right)$, respectively. An estimate of the relationship between survivorship of haploid males and females, $a$, came from previously published data showing that female mortality exceeded male mortality by a factor of 1.1, regardless of clutch size (Ode et al., 1996).

We estimated the range of potential diploid male survivorship from the fraction of matings that produced only males. This was possible because the twoallele lines used in the crosses were (by definition) producing diploid males, some fraction of which were presumably surviving to adulthood. Between 75 and $95 \%$ of these surviving diploid males are sterile, with the balance being able to sire only a few triploid daughters (Bostian, 1936; Whiting, 1961). Females in our experimental crosses producing all-male broods were presumably either unmated (Petters et al., 1985; Ode et al., 1995), mated to haploid males that did not pass sperm, or mated to one of these surviving diploid males. The maximum rate of diploid male survivorship can therefore be calculated by attributing the failure to produce daughters to diploid male mates in every such cross. This is likely to overestimate diploid male survivorship and leads to a conservative test because previous studies indicated that most females producing all-male progeny did so because they did not mate (Petters et al., 1985; Ode et al., 1995; Guertin et al., 1996). Under the very conservative assumption that all matings failing to produce daughters are attributable to matings with diploid males, the likelihood that a cross will produce only sons is the product of the probability of a given male being diploid and the rate of diploid male survivorship. The maximum rate of diploid male survivorship, $\delta_{\max }$, can therefore be expressed as $p /\{0.5 f /[0.5 f+(1-f)]\}$, where $p$ is the observed fraction of matings that produce only males. The quantity $p$ was obtained from our experimental crosses, and $\delta_{\max }$ was estimated using a fertilization rate of 0.5 , which is lower than the average fertilization rate observed in laboratory experiments (Horn, 1943; Whiting, 1943; Petters \& Mettus, 1980; Antolin et al., 1995; Heimpel et al., 1997; Ode et al., 1997) and leads to a conservative estimate of $\delta_{\max }$ with respect to the significance tests.

Of the 691 crosses conducted, $54(7.8 \%)$ produced only male offspring. The production of only male offspring was affected neither by cross type $\left(\chi_{2}^{2}=3.0\right.$, $P>0.2)$ nor the population from which either parent was taken (mother's population: $\chi_{4}^{2}=1.3, P>0.5$; father's population: $\chi_{4}^{2}=7.0, P>0.1$ ). Our estimate of $p$, the proportion of matings producing only males, is therefore 0.078 , and, using 0.5 as a value for the fertilization rate $f$, leads to a maximum rate of diploid survival, $\delta_{\max }$, of 0.234 . Our low, central and high estimates for $\delta$ were therefore $0,0.10$ and 0.234 . The central estimate is an average value cited in the literature for B. hebetor (Horn, 1943; Whiting, 1943; Grosch, 1945; Petters \& Mettus, 1980). 


\section{Allele identification}

Alleles were identified by confirming that given matings were among individuals that did not share a sex allele. The criterion for different alleles was that each of the nine goodness-of-fit tests lead to rejection of the null hypothesis for a given cross. For this to be true, the lowest $G$-value $\left(G_{\min }\right)$ must be greater than the critical $G$-value for a given level of confidence (Sokal \& Rohlf, 1981). We used a probability level of $\alpha=0.01$, so that any set of nine goodness-of-fit tests that yielded a $G_{\min }$ of greater than $6.63\left(=G_{\text {crit }}\right)$ indicated significant differences in egg mortality and secondary sex ratio from those expected if sex alleles were shared under the conditions described by any of the nine tests. Alleles were distinguished by scoring $G_{\min }$ for each of the five replicate matings for a given cross. If $G_{\min }$ for every replicate mating was greater than $G_{\text {crit }}$, we concluded that no alleles were shared among the lines. If none of the replicate matings produced $G_{\min }$ greater than $G_{\text {crit }}$, there was no evidence for differences in alleles among the two lines, and the data were considered consistent with both alleles being shared among the lines. Finally, if one or more $G_{\min }$ were greater than $G_{\text {crit }}$, with the balance less than or equal to $G_{\text {crit }}$, we concluded that a single allele was shared by the two lines. Alleles were counted by comparing the numbers of replicate matings between the different lines that produced $G_{\min }>G_{\text {crit }}$.

\section{Results}

Both egg mortality and secondary sex ratio were highest in within-line (control) crosses, intermediate in crosses carried out within populations but between lines, and lowest in between-population crosses (Table 1; nested ANOVA on arcsine-transformed values: $F_{2,17}=19.9$, $P<0.0001$ for egg mortality; $F_{2,17}=12.4$, $P<0.001$ for sex ratio). Egg mortality and sex ratio also differed significantly between populations in the within-population crosses (Table 2). For the 10 classes of between-population crosses, we found significant differences in the egg mortality rate $\left(F_{9,253}=3.2\right.$; $P<0.01)$, but not in the secondary sex ratio $\left(F_{9,253}=1.6 ; P>0.1\right)($ Fig. 1$)$.

\section{Control (within-line) crosses}

In matings within two-allele lines, we expect none of the nine goodness-of-fit tests to lead to rejection of the null hypothesis in any of the crosses. Figure 2 shows $G_{\min }$ as a function of egg hatch rate and secondary sex ratio for the within-line crosses. Out of 123 crosses, five had $G_{\min }>G_{\text {crit }}$. These five crosses came from three lines (two each from one of the CA and INS lines and one
Table 1 Egg mortality and secondary sex ratio (proportion of adult offspring that were male) in Bracon hebetor from crosses within lines (control crosses), crosses within populations but between lines, and crosses between populations. See text for statistical analysis

\begin{tabular}{lccc}
\hline Cross type & $N$ & $\begin{array}{c}\text { Egg mortality } \\
\text { (SEM) }\end{array}$ & $\begin{array}{c}\text { Sex ratio } \\
\text { (SEM) }\end{array}$ \\
\hline Control (within lines) & 123 & 0.347 & 0.547 \\
& & $(0.011)$ & $(0.014)$ \\
Within populations & 251 & 0.178 & 0.411 \\
& & $(0.010)$ & $(0.010)$ \\
Between populations & 263 & 0.123 & 0.356 \\
& & $(0.006)$ & $(0.007)$ \\
\hline
\end{tabular}

from one of the KS lines), indicating that, of the 25 isofemale lines in the study, three contained three sex alleles. We suspect that the additional alleles were introduced by contamination from other isofemale lines, but cannot rule out the possibility that they arose by mutation.

\section{Within-population (between-line crosses)}

Minimum $G$-values are shown as a function of egg hatch and sex ratio for all of the within-population crosses in Fig. 3. The number of alleles detected in the five populations of the study was three or four (WKE), four (MID), six (KS), nine (CA) and five or six (INS)

Table 2 Egg mortality and secondary sex ratio (proportion of adult offspring that were male) from within-population between-line crosses for each of the five populations of Bracon hebetor

\begin{tabular}{lccc}
\hline & $N$ & $\begin{array}{c}\text { Egg } \\
\text { mortality } \\
\text { (SEM) }\end{array}$ & $\begin{array}{c}\text { Proportion } \\
\text { males } \\
\text { (SEM) }\end{array}$ \\
\hline $\begin{array}{l}\text { Waunakee, WI } \\
\text { (WKE) }\end{array}$ & 50 & 0.229 & 0.448 \\
Middleton, WI & 50 & $(0.022)$ & $(0.023)$ \\
(MID) & & 0.262 & 0.502 \\
Kansas (KS) & 51 & $(0.020)$ & $(0.024)$ \\
& & 0.173 & 0.336 \\
California (CA) & 50 & $(0.023)$ & $(0.017)$ \\
& & 0.067 & 0.342 \\
Insectary (INS) & 50 & $(0.009)$ & $(0.018)$ \\
& & 0.161 & 0.428 \\
$F_{4,246}$ & & $(0.017)$ & $(0.020)$ \\
\hline
\end{tabular}

(C) The Genetical Society of Great Britain, Heredity, 82, 282-291. 
Fig. 1 Egg mortality (solid bars) and secondary sex ratio (proportion of adult offspring that were male; hatched bars) in Bracon hebetor produced by the 10 classes of between-population crosses (crosses between specific isofemale lines pooled). Vertical lines are standard errors of the means and numbers are the number of matings carried out per population combination. See text for statistical analysis.

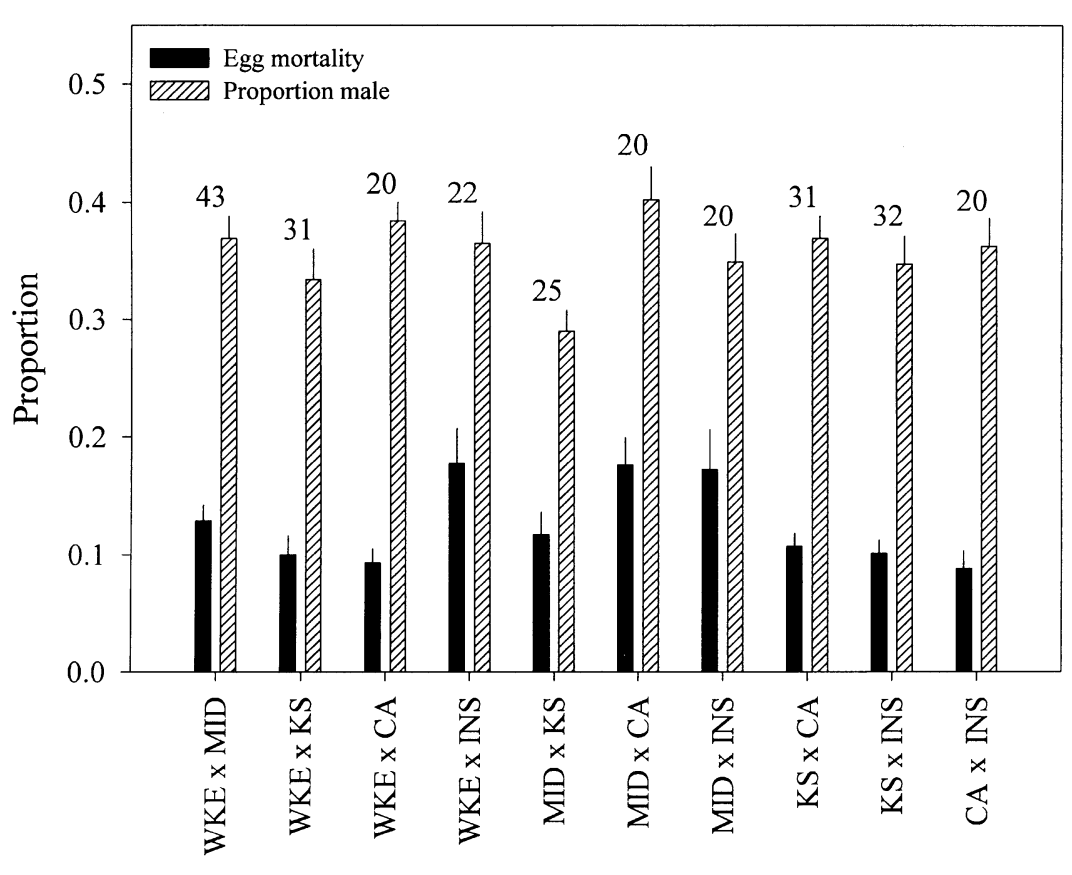

Cross
(Table 3). We estimated the predicted number of alleles, $n$, for each population using a simulation approach by randomly sampling five combinations of two alleles for allele diversities ranging between two and 100. Simulations were run 10000 times for each value of $n$ and we calculated the mean number of sex alleles sampled, along with the $95 \%$ confidence interval using standard

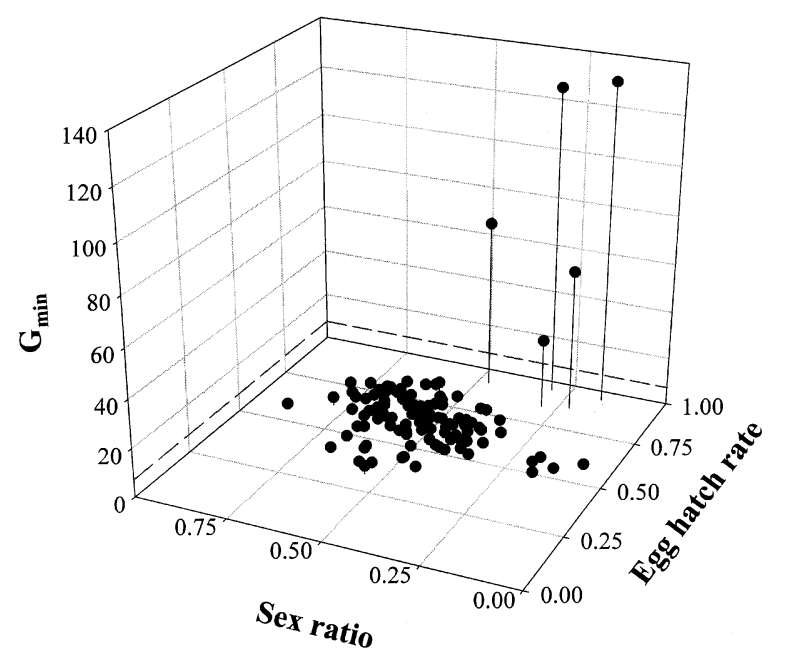

Fig. 2 Minimum $G$-values $\left(G_{\min }\right)$ for each of the control (within-line) crosses of Bracon hebetor as a function of the sex ratio (proportion of adult offspring that were male) and egg hatch rate. The dotted line shows the critical $G$-value (6.63) above which the null hypothesis of shared sex alleles can be rejected. bootstrapping methods (Efron \& Tibshirani, 1993) (Table 3).

\section{Between-population crosses}

Between-population crosses were subsampled by using two lines from each population for the crosses. The within-population crosses showed that alleles were not shared between these lines within every population except for WKE, where the lines shared one allele. We identified 12 sex alleles from these crosses (Table 4). The four alleles sampled from the CA population were not found in any of the other lines tested, and single unique alleles were found in the MID, KS and INS populations. One allele was found exclusively in two populations (allele $c$ in WKE and MID; Table 4). Data consistent with allele-sharing among the WKE, THI, KS and INS populations were found for one of the alleles ( $a$ or $b$ ) (Table 4).

We can estimate the number of alleles that were missed because of subsampling the between-population crosses, $K$, from the number of alleles identified from each population, $A_{i}$, the number of alleles sampled from each population, $S_{i}$, and the number of alleles that were shared by $q$ populations, $k_{q}$ :

$$
K=\sum_{i=1}^{5}\left\{\left[\begin{array}{ll}
A_{i} & S_{i}
\end{array}\right]\left[1 \quad\left(\sum_{q=1}^{5} k_{q, i}\right) / \sum_{i=1}^{5} S_{i}\right]\right\} .
$$



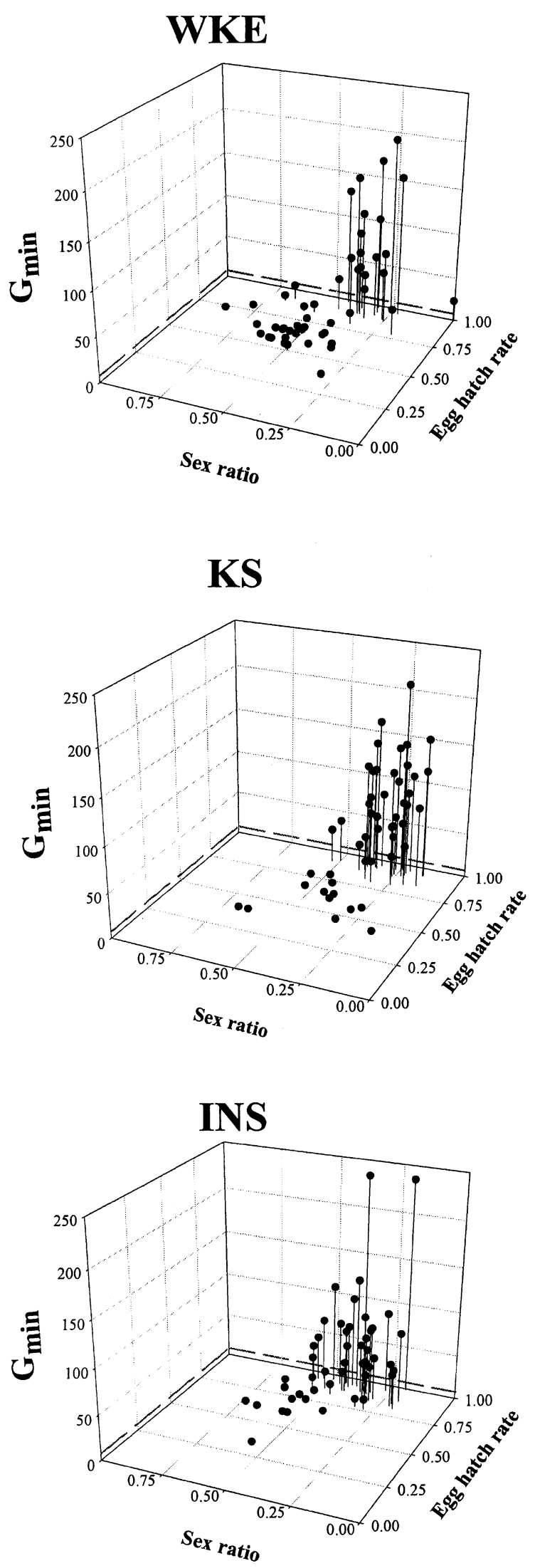
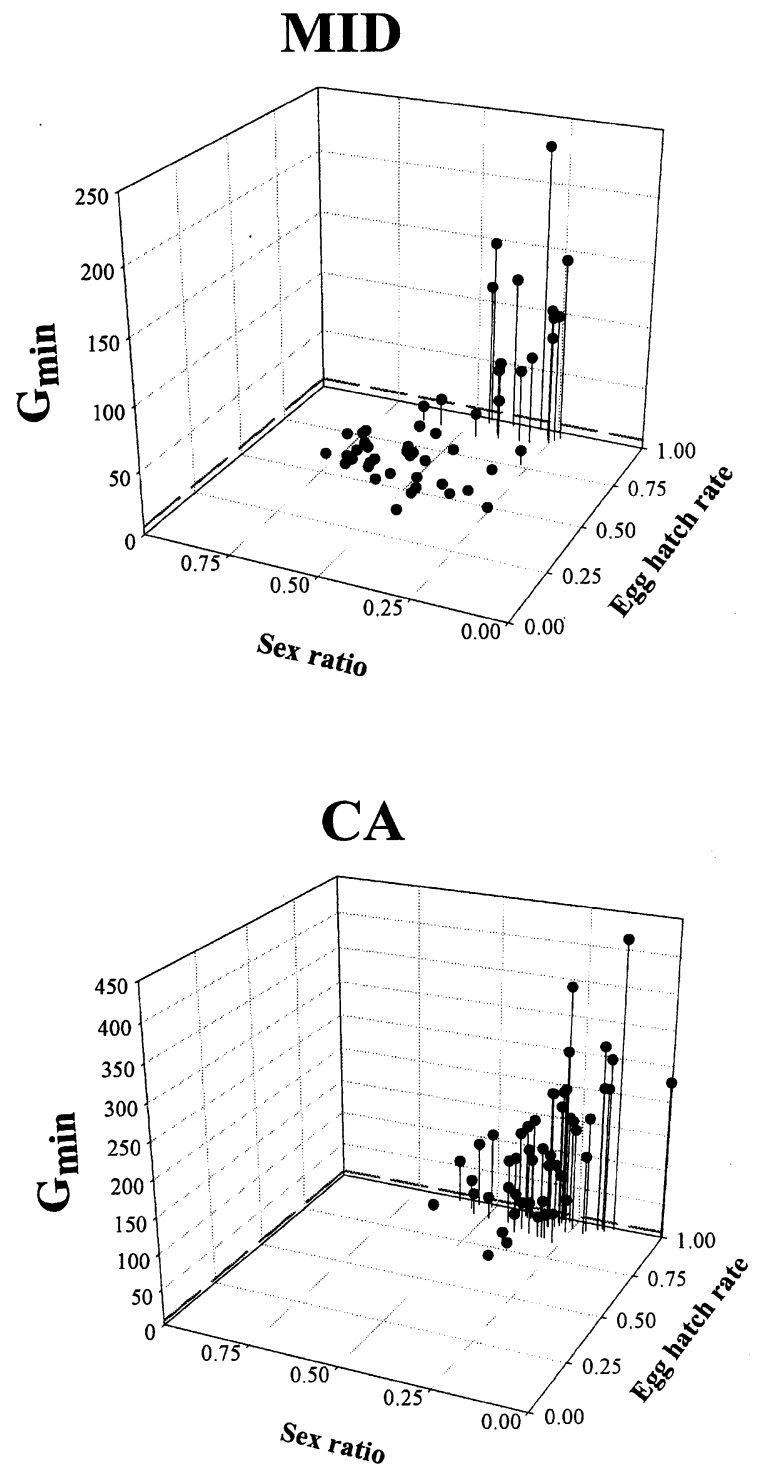

Fig. 3 Minimum $G$-values $\left(G_{\min }\right)$ for each of the withinpopulation (between-line) crosses of Bracon hebetor as a function of the sex ratio (proportion of adult offspring that were male) and egg hatch rate for each of the five populations in the study. The dotted lines in each of the plots show the critical $G$-value (6.63) above which the null hypothesis of shared sex alleles can be rejected. 
Table 3 Number of combinations of isofemale lines from each of the five populations of Bracon hebetor in which none, all, or some of the five replicate crosses were consistent with different sex alleles (i.e. $G_{\min }>G_{\text {crit }}$ ). Also, numbers of sex alleles identified from each population, and the estimated number of alleles present in each population, $n$, with $95 \%$ confidence intervals from a bootstrapped data set

\begin{tabular}{|c|c|c|c|c|c|c|}
\hline Population & \multicolumn{3}{|c|}{$\begin{array}{l}\text { Combinations of isofemale lines } \\
\text { showing evidence of different alleles }\end{array}$} & $\begin{array}{c}\text { Number of } \\
\text { alleles } \\
\text { identified }\end{array}$ & $\begin{array}{c}\text { Estimated } \\
n\end{array}$ & $\begin{array}{c}95 \% \\
\text { confidence } \\
\text { interval for } n\end{array}$ \\
\hline WKE & 2 & 8 & 0 & 3 or 4 & 3 or 4 & $3-7$ \\
\hline MID & 3 & 5 & 2 & 4 & 4 & $4-7$ \\
\hline $\mathrm{CA}$ & 0 & 1 & 9 & 9 & 38 & $12-100+$ \\
\hline INS & 0 & 7 & 3 & 5 or 6 & 6 or 8 & 5-18 \\
\hline
\end{tabular}

This extrapolation leads to an estimate of $K=8.3$ sex alleles missed by our subsampling procedure. The estimate for the total number of alleles in our study can therefore be increased from 12 to 20 .

\section{Discussion}

Previous work identified nine alleles distributed among six $B$. hebetor populations. In our study, we have sampled more extensively and have identified 12 alleles, and extrapolation leads to a total of 20 present in our isofemale lines. However, as each isofemale line was initiated with a single female and only five isofemale lines were used per population, this estimate must be viewed as a minimum value. The number of alleles sampled per population (again, a minimum estimate) ranged from three or four to nine, and there were clear differences in the allele profiles of each population. All of the field populations from which we sampled contained six or more alleles, suggesting a relatively weak sex determination load (Stouthamer et al., 1992).
Some of the differences in allele diversity among sites may be related to the structure of the populations from which they were sampled. For instance, the fact that the greatest number of sex alleles (9) was found at the California site is perhaps not surprising, given its commercial management. Figs originating at a number of orchards that support populations of $B$. hebetor are stored at this site (J. Johnson, pers. comm.). The California population therefore represents a sink that receives immigrants from multiple sites. The Kansas site, on the other hand, consisted of a single on-farm silo that was reportedly emptied and cleaned annually and received wheat directly from the field. The source of $B$. hebetor at this site is unknown and it is unclear whether populations persist throughout the year. Presumably, the population is either initiated each year as a founder population or persists in the grain tailings as small populations from year to year. In either case, one would expect relatively low sex allele diversity. The number of sex alleles that we identified (6) represents lower diversity than the California site (as expected),

Table 4 Number of replicate crosses (out of at least five) which were consistent with different sex alleles (i.e. $G_{\text {min }}>G_{\text {crit }}$ ) for the between-population crosses of Bracon hebetor

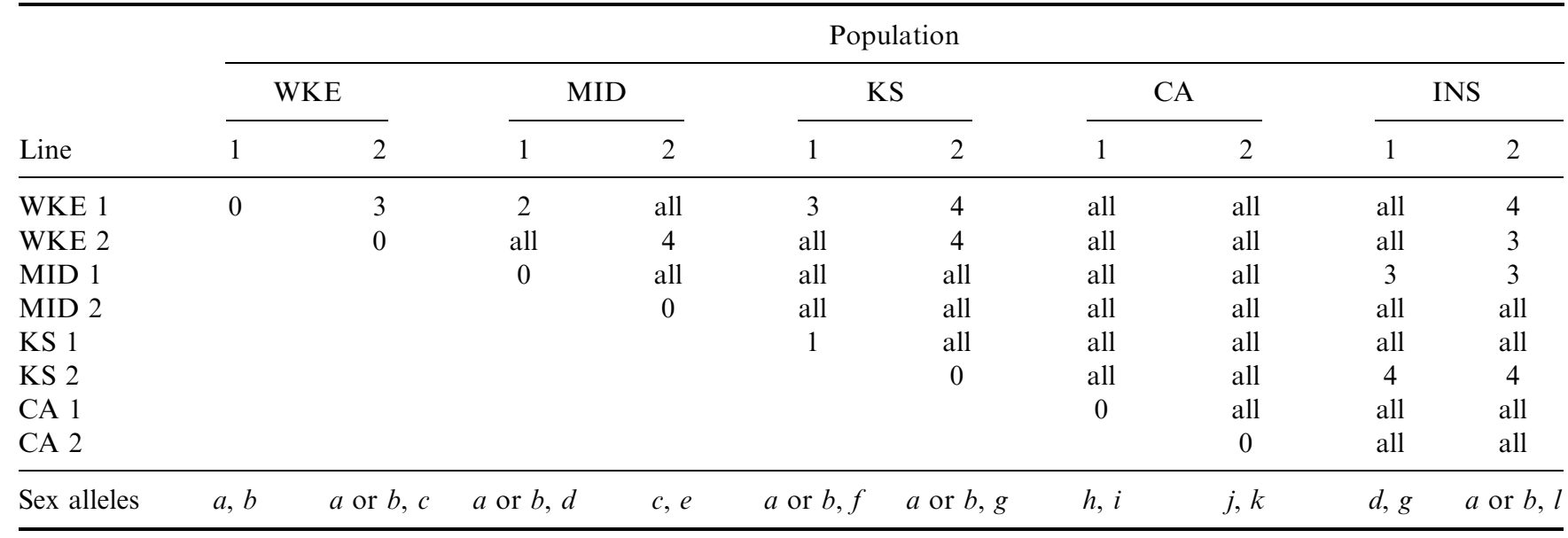

(C) The Genetical Society of Great Britain, Heredity, 82, 282-291. 
but was higher than anticipated given that these six alleles were found in a random sample of only five females.

Low allele diversity was found in the two Wisconsin populations, but this almost certainly resulted from the relatively long time this population was maintained as relatively small cultures in the laboratory. Supporting this conclusion is our own historical data on the secondary sex ratio produced by the MID population. Females from this population produced a female-biased sex ratio upon collection from the field in 1991 (0.34 proportion males; Ode et al., 1997) whereas withinpopulation crosses carried out here produced a sex ratio of 0.5 (Table 2). In contrast, the five or six alleles identified in the commercial insectary population (INS) suggests that using rearing cages with large populations ( $>700$ individuals per cage in this case) is a sound strategy for maintaining relatively high sex allele diversity during mass-rearing (Stouthamer et al., 1992).

The between-population crosses were consistent with both shared and disparate alleles among populations (Table 4). It is possible, however, that accidental contamination of isofemale lines with individuals from different populations increased our estimate of allele sharing among populations, and these data must be viewed with caution. The danger of contamination was probably greatest between the WKE and MID populations, because they had been cultured side by side for 3 years prior to the experiment. Our estimates for disparate alleles are more resistant to contamination effects, however, because alleles cannot be lost in their entirety from two-allele isofemale lines (Cook, 1993a). At high rates of contamination, it is conceivable that the original alleles could become relatively rare and therefore not be detected in five replicate crosses. However, our control (within-line) crosses demonstrated a relatively low rate of contamination during the course of the study: three isofemale lines out of 25 appeared to harbour three (instead of two) alleles (Fig. 2). Also, our statistical test was conservative with respect to sex alleles not being shared, so that our conclusions of alleles not being shared among two given lines are more robust than conclusions that they are shared.

Our finding that different populations of $B$. hebetor harbour different sex alleles indicates that interpopulation dispersal could restore sex allele diversity in populations that are depauperate in sex alleles. Given the close association of $B$. hebetor with moths attacking stored grain, fruit and nuts, most dispersal is likely to be passive and mediated by human activities, although there are reports of $B$. hebetor from field habitats in the old world (e.g. Puttarudriah \& Channa Basavanna, 1956; Gerling, 1971; Youm \& Gilstrap, 1993). The extent to which active dispersal between populations contributes to within-population sex allele diversity remains to be determined.

\section{Acknowledgements}

We thank Drs Barry Dover and Judy Johnson for their aid in locating field sites. For laboratory assistance, we thank Carrie Graf, Miri Halevy and Jonathan Lundgren. For discussions and advice on the manuscript, we thank Charles Baer, Paul Ode and two anonymous reviewers. This research was supported in part by U.S.D.A. Grant 9402217 to M.F.A. and M.R.S. and U.S.D.A. Postdoctoral Fellowship 9502315 to G.E.H.

\section{References}

ANTOLIN, M. F. AND STRAND, M. R. 1992. Mating system of Bracon hebetor (Say) (Hymenoptera: Braconidae). Ecol. Entomol., 17, 1-7.

ANTOLIN, M. F., ODE, P. J. AND STRAND, M. R. 1995. Variable sex ratios and ovicide in an outbreeding parasitic wasp. Anim. Behav., 49, 589-600.

BENSON, J. F. 1973. Intraspecific competition in the population dynamics of Bracon hebetor Say. J. Anim. Ecol., 42, 105124.

Bostian, C. H. 1936. Fecundity of triploid females in Habrobracon juglandis. Am. Nat., 70, 40-41.

BROWER, J. H., SMITH, L., VAIL, P. V. AND FLINN, P. W. 1996. Biological control. In: Subramanyam, B. and Hagstrum, D.W. (eds) Integrated Management of Insects in Stored Products, pp. 223-286. Marcel Dekker, New York.

BULl, J. J. 1983. Evolution of Sex Determining Mechanisms. Benjamin/Cummings, Menlo Park, CA.

COOK, J. M. 1993a. Inbred lines as reservoirs of sex alleles in parasitoid rearing programs. Envir. Entomol., 22, 12131216.

COOK, J. M. 1993b. Sex determination in the Hymenoptera: a review of models and evidence. Heredity, 71, 421-435.

COOK, J. M. AND CROZIER, R. H. 1995. Sex determination and population biology in the Hymenoptera. Trends Ecol. Evol., 10, 281-286.

COOK, J. M., RIVERO-LYNCH, A. P. AND GODFRAY, H. C. J. 1994. Sex ratio and foundress number in the parasitoid wasp Bracon hebetor. Anim. Behav., 47, 687-696.

EFRON, B. AND TIBSHIRANI, R. J. 1993. An Introduction to the Bootstrap. Chapman \& Hall, New York.

GERLING, D. 1971. Occurrence, abundance, and efficiency of some local parasitoids attacking Spodoptera littoralis (Lepidoptera: Noctuidae) in selected cotton fields in Israel. Ann. Entomol. Soc. Am., 64, 492-499.

GROSCH, D. S. 1945. The relation of cell size and organ size to mortality in Habrobracon. Growth, 9, 1-17.

GUERTIN, D. S., ODE, P. J., STRAND, M. R. AND ANTOLIN, M. F. 1996. Host-searching and mating in an outbreeding parasitoid wasp. Ecol. Entomol., 21, 27-33.

hagstrum, D. W. AND Smittle, B. J. 1978. Host utilization by Bracon hebetor. Envir. Entomol., 7, 596-600. 
HEIMPEL, G. E., ANTOLIN, M. F., FRANQUI, R. A. AND STRAND, M. R. 1997. Reproductive isolation and genetic divergence between two 'strains' of Bracon hebetor (Hymenoptera: Braconidae). Biol. Control, 9, 149-156.

HORN, A. B. 1943. Proof for multiple allelism of sex-differentiating factors in Habrobracon. Am. Nat., 77, 539-550.

JOHNSON, w. G. 1895. A new parasite of the Mediterranean flour moth. Entomol. News, 6, 324-325.

KROMBEIN, K. V., HURD, P. D., JR, SMITH, D. R. AND BURKS, B. D. 1979. Catalog of Hymenoptera in America North of Mexico. Smithsonian Institution Press, Washington, DC.

ODE, P. J., ANTOLIN, M. F. AND STRAND, M. R. 1995. Brood-mate avoidance in the parasitic wasp Bracon hebetor Say. Anim. Behav., 49, 1239-1248.

ODE, P. J., ANTOLIN, M. F. AND STRAND, M. R. 1996. Sex allocation and sexual asymmetries in intra-brood competition in the parasitic wasp Bracon hebetor. J. Anim. Ecol., 65, 690-700.

ODE, P. J., ANTOlin, M. F. AND STRAND, M. R. 1997. Constrained oviposition and female-biased sex allocation in a parasitic wasp. Oecologia, 109, 547-555.

OWEN, R. E. AND PACKER, L. 1994. Estimation of the proportion of diploid males in populations of Hymenoptera. Heredity, 72, 219-227.

PETTERS, R. M. AND MeTtus, R. v. 1980. Decreased diploid male viability in the parasitic wasp, Bracon hebetor. J. Hered., 71, 353-356.

PETTERS, R. M., KENDALl, M. E., TAYLOR, R. A. J. AND METTUS, R. E. 1985. Time required for mating and the degree of genetic relatedness in the parasitoid wasp Bracon hebetor
Say (Hymenoptera: Braconidae). Mels. Entomol. Ser., 35, 21-27.

POTTS, W. K. AND WAKELAND, E. K. 1993. Evolution of MHC genetic diversity: a tale of incest, pestilence and sexual preference. Trends Genet., 9, 408-412.

PUTTARUdRIAH, M. AND CHANNA BASAVANNA, G. P. 1956. A study on the identity of Bracon hebetor Say and Bracon brevicornis Wesmael. Bull. Ent. Res., 47, 183-191.

SAY, T. 1836. Descriptions of new species of North American Hymenoptera, and observations on some already described. Boston J. Nat. Hist., 1, 209-305.

SOKAL, R. R. and ROHLF, F. J. 1981. Biometry, 2nd edn. W.H. Freeman \& Co., New York.

STOUTHAMER, R., LUCK, R. F. AND WERREN, J. H. 1992. Genetics of sex determination and the improvement of biological control using parasitoids. Envir. Entomol., 21, 427-435.

WERREN, J. H. 1993. The evolution of inbreeding in haplodiploid organisms. In: Thornhill, N. W. (ed.) The Natural History of Inbreeding and Outbreeding, pp. 42-59. University of Chicago Press, Chicago, IL.

Whiting, A. R. 1961. Genetics of Habrobracon. Adv. Genet., 10, 295-348.

WHitiNG, P. W. 1943. Multiple alleles in complementary sex determination of Habrobracon. Genetics, 24, 110-111.

YOUM, O. AND GILSTRAP, F. E. 1993. Population dynamics and parasitism of Coniesta (= Haimbachia) ignefusalis, Sesamia calamistis, and Heliocheilus albipunctella in millet monoculture. Ins. Sci. Appl., 14, 419-426. 